\title{
El concepto de competencia y la educación como el cuidado de sí mismo*
}

\section{The concept of competence and education as self-care}

\author{
"Nosce Te Ipsum"** \\ Olga Elvira Acosta $\in m \in I^{* * *}$
}

RECIBIDO: 24 DE SEPTIEMBRE DE 2012 • ACEPTADO: 26 DE OCTUBRE DE 2012

Resumen. El concepto de competencia es esencial para entender las dinámicas educativas actuales. Su importancia se deja ver no solo desde el punto de vista académico, sino político y económico. En este trabajo se pretende analizar las principales tendencias que permiten comprender este concepto. De la misma forma el propósito es hacer una evaluación crítica de la manera como generalmente se le entiende. Para esto se utilizará el concepto de "cuidarse a sí mismo» que se retoma de la filosofía de Michel Foucault. Al final, se pretende

* Artículo de revisión de tema que analiza el concepto de competencia desde la perspectiva del «cuidado de sí mismo», expuesto por Michel Foucault.

** Traducción latina de la Inscripción que los antiguos griegos encontraban en la entrada del oráculo de Delfos.

*** Especialista en Gestión Educativa, Universidad de Pamplona; Magister en Ciencias de la Educación y Especialista en Administración Educativa, Universidad de San Buenaventura; Candidata a Doctora en Conocimiento y Cultura en América Latina en el Instituto de Pensamiento y Cultura en américa Latina ( IPECAL). Rectora de la Institución Educativa Olga González Arraut; consultora de la Agencia de Cooperación Alemana (GIZ), Proyecto PRODEMUJER; Consultora de la Fundación Pies Descalzos del proyecto: La escuela como posibilidad de vida para niñas, niños y jóvenes en situación de desplazamiento y vulnerabilidad. Directora de la Escuela de Gobierno y Liderazgo de la Alcaldía Distrital Cartagena y docente de la Universidad de San Buenaventura, Cartagena. E-mail: olacoamel@hotmail.com 
mostrar una imagen de la educación que nos permite entenderla como el proceso por medio del cual el individuo se encuentra a sí mismo.

Palabras clave. Competencia, educación, cuidado de sí, pedagogía.

Abstract. The concept of competition is essential to understand the current educational dynamics. Its importance is evident not only from the academic point of view, also political and economic. This work intends to analyze the main trends that allow us to understand this concept. In the same way the purpose is to make a critical assessment of the way how generally understood you. This will use the concept of "look after yourself» which takes up the philosophy of Michel Foucault. At the end, is intended to show a picture of education that allows us to understand it as the process through which the individual finds himself.

Keywords. Competition, education, self-care, pedagogy.

\section{Introducción}

En la pedagogía y en la política educativa actual el concepto de competencia es esencial. Podemos afirmar que es desde este concepto que se organizan todos los otros dispositivos institucionales y académicos propios de la actividad educativa. Así, comprender este concepto es esencial para entender los diversos elementos en los que, como maestros y administradores educativos, nos vemos inmersos a diario.

Sin embargo, el problema es que no hay una definición de competencia que sea aceptada universalmente, y no es claro igualmente el origen mismo del concepto. Es necesario después de todo que nosotros partamos de al menos una descripción de lo que es "competencia». Según el paradigma chosmkyano (Ruiz, 2004), la competencia se define como una capacidad autónoma del individuo. Esta capacidad se expresa en la posibilidad de creación y producción, actuación y transformación, que se manifiesta en el intercambio social, en el contacto con las y los otros. El concepto de competencia parece abordar la diferencia entre un conocimiento puramente teórico, y un conocimiento que es aplicado en un contexto, por una persona y en relación a una situación que puede involucrar a otros. La idea del sujeto que realiza la acción entonces toma una posición crucial. El concepto de competencia también hace alusión 
a una habilidad que puede ser utilizada en diferentes contextos, y por tanto, que implica un elemento de creatividad y de adaptación para afrontar situaciones imprevistas.

Ahora bien, en la medida que el concepto de competencia es utilizado como una herramienta de definición y referencia de procesos educativos institucionales es posible reconocer diferentes perspectivas de análisis y de interpretación de este concepto más o menos general.

Desde el punto de vista político y económico la competencia es vista en términos principalmente (no únicamente) como un «saber cómo». Desde el punto de vista del conocimiento académico y disciplinar es percibido principalmente como un «saber qué». Desde el punto de vista de la filosofía la competencia es vista como un tipo de «conocimiento de sí mismo». Y por último desde el punto de vista de de la actividad del maestro se ve como un conocimiento reflexivo englobante. De estas perspectivas la primera y la penúltima requieren tal vez un tratamiento más detallado. Aquí trataremos la primera y dejaremos el apartado siguiente para la tendencia filosófica.

\section{El concepto de competencia y las dinámicas políticas y económicas educativas}

Si analizamos el concepto de competencia a la luz de la tendencia política y económica tenemos una perspectiva muy diferente de acercarnos a las competencias. La descripción aquí se centra en los detalles pragmáticos del concepto y hace énfasis en los resultados prácticos de un hacer en contexto. Esta forma de abordar las competencias se centra en la eficacia práctica y económica de un saber y por tanto se enmarca en un contexto económico y político que se interesa por la productividad laboral y por las necesidades del mercado. Esta perspectiva es importante en la medida que las políticas educativas, sobre todo en Colombia, se centran en ella hasta el punto de transformar esta tendencia en el modelo para juzgar las otras. Esto es así pues aquí estamos interesados en la educación en un contexto político y económico globalizado, donde las exigencias de los individuos son definidas a partir de criterios operativos.

Hasta qué punto las otras tendencias pueden servir de contrapeso a esta tendencia es algo que deberemos analizar más adelante. Por el momento es importante intentar comprender la manera como esta disposición se

ITINERARIO EDUCATIVO • ISSN OILI-C753 • AÑO XXVI, N. ${ }^{\circ} 60$ • ENERO - JUNIO DE ZOIL • P. I93-ZOI 
conecta directamente con la política educativa de calidad, sobre todo en el caso colombiano. Esto es importante pues la influencia del enfoque de las competencias en educación es conectando de manera aún más estrecha que antes la educación con el sector laboral (Ministerio de Educación Nacional, 2005). Hasta el punto que el sector laboral hace presencia en las discusiones, análisis, revisiones y transformaciones de la educación. Esta unidad de criterios entre el sector laboral y el educativo va hasta el punto de reducir la definición de competencia al ámbito únicamente del «saber hacer». Así, la competencia se define como "un saber hacer flexible que puede actualizarse en distintos contextos, es decir, como la capacidad de usar los conocimientos en situaciones» (Ministerio de Educación Nacional, 2006). Esto obviamente no implica una reducción total, se sigue teniendo en cuenta que este saber hacer se encuentra contextualizado en unas consideraciones éticas, sociales y políticas. Pero estos aspectos parecen reducir su importancia teniendo en cuenta la definición ofrecida.

El propósito entonces es crear una especie de circuito de retroalimentación que inicie con un currículo basado en las competencias, a partir de allí pase a la creación de unos estándares básicos para su evaluación, de allí a su evaluación efectiva, es decir basados en la evidencia de si el sujeto puede insertarse y reinsertarse varias veces en la sociedad como lo plantea Zemelman (2010). Los resultados de esta evaluación serán usados para determinar planes de mejoramiento que puedan utilizarse para el fortalecimiento de los currículos (Ministerio de Educación Nacional, 2006) pero también del sujeto. Así, las competencias y sus estándares, definidos a partir de un «saber hacer», son la guía fundamental no solo para el diseño de currículos, de los proyectos escolares y del trabajo pedagógico en el aula, sino también para la evaluación institucional local y nacional de tales currículos y proyectos. Es por esta razón que la evaluación es tan importante, pues ella se encuentra en el corazón mismo del circuito que termina con el mejoramiento de los currículos ya que los influye de manera transversal y provee criterios para evaluar la pertinencia del currículo en su totalidad (Salas, s.f.).

\section{Las competencias desde un punto de vista filosófico: Foucault y el conocimiento de sí mismo}

Desde el punto de vista filosófico la filosofía tradicional de corte aristotélico tomista define la competencia a partir de la noción de potencia. 
La potencia es un «poder para ejercer una transformación en un objeto o disposición para poder llegar a ser algo» (Arias, 2009, p.67). La potencia es vista no sólo como la capacidad de hacer algo, sino también como la capacidad para llegar a ser otra cosa a través de lo que ya uno es. La filosofía parte de una consideración de la esencia humana y a partir de allí muestra la conducta del ser humano como el desarrollo de una potencialidad que es fruto de esa esencia. Desde esta perspectiva, la potencia es vista como un ámbito donde la autonomía del sujeto se desarrolla en el tiempo. La competencia entonces da fruto a un tipo de autoconocimiento esencial que permite el desarrollo de capacidades para la mejor comprensión de sí mismo y del entorno.

Sin embargo, la tendencia filosófica se puede evaluar de manera diferente a la que hemos visto. La filosofía aristotélico-tomista tiene una importancia que es imposible de negar, pero desde entonces han aparecido otras filosofías y es posible que estas tengan otras riquezas que sirvan de aporte a la discusión de las competencias. De la misma forma, parte de una idea del ser humano a partir de una esencia y por eso mismo parece ya predefinir sus potencialidades. Por último, la concepción de conocimiento de uno mismo predetermina cierto tipo de relación del hombre consigo mismo, una relación que enmascara otras relaciones entre el yo y sí mismo que evaden consideraciones intelectuales y se presentan más con la riqueza de lo ético y existencial.

Para entender esto es importante hacer una evaluación crítica de la noción de conocimiento de uno mismo, que es la idea fundamental de la noción de competencia entendida filosóficamente, a partir de las reflexiones que Michel Foucault elaboró en sus cursos en el colegio de Francia entre 1981 y 1982. Estas reflexiones nos servirán para hacer una evaluación crítica de las tendencias educativas y de la preponderancia del saber cómo en la definición de competencia.

Con el conocimiento de uno mismo se busca una relación del hombre consigo mismo basada en el autocontrol y la autorregulación (Arias, 2009). Esta relación se ha acentuado sobre todo con el cristianismo y la noción platónica de conocimiento (Foucault, 1994, p. 26). Sin embargo, según Foucault, la idea de conocerse a sí mismo es un simple caso particular de un concepto mucho más amplio que ha sido entendida en la actualidad solamente a través de su caso particular. Este concepto es según Foucault el concepto de cuidado «epimeleia» (Foucault, 1994, p. 
33). Cuidarse a uno mismo «epimeleia sui» siempre acompaña, en la antigüedad griega al consejo del oráculo de Delfos: cuídate a ti mismo. Existen múltiples maneras de entender el cuidado de sí mismo, pero fundamentalmente Foucault entiende por cuidarse a sí mismo el cuidado de su alma. El alma entendida en lo general, como aquello que diferencia a un ser humano de otros. Desde esta perspectiva por ejemplo el médico que se cura a sí mismo no es un caso de cuidado de sí, pues éste se preocupa de su cuerpo, no de sí mismo (Foucault, 1994, p. 48).

Los conceptos de autoconocimiento y autocuidado remiten a su vez a una concepción de la actividad pedagógica en tanto regulan el acceso del sujeto a la verdad (Foucault, 1994). Pero también, como bien afirma Foucault, esta idea del cuidado de sí apunta necesariamente a una relación pedagógica esencial que es importante analizar:

No existe preocupación por uno mismo sin la presencia de un maestro, pero lo que define la posición del maestro es que aquello de lo que él se ocupa es precisamente el cuidado que pueda tener sobre sí mismo aquel a quien él sirve de guía. El maestro es quien se cuida del cuidado del sujeto respecto a sí mismo y quien encuentra en el amor que tiene por su discípulo la posibilidad de-ocuparse del cuidado que el discípulo tiene de sí mismo. Al amar de forma desinteresada al joven discípulo, el maest es el principio y el modelo del cuidado de uno mismo que el joven debe de tener de sí en tanto que sujeto (p. 49).

Esta maravillosa cita resume toda una filosofía de la pedagogía apoyada en la idea de cuidado de sí. La labor del maestro es ofrecerle al alumno los medios de encontrase a sí mismo por medio del cuidado de sí mismo. El concepto fundamental no es el de «saber qué», ni el de «saber cómo», sino el del amor, amor a sí mismo y al conocimiento.

Amor a sí mismo no debe entenderse como una forma de egoísmo o interés individual, todo lo contrario, cuidar de uno mismo es imposible sin el cuidar de los otros. El cuidado de sí implica:

...relaciones complejas con los otros, en la medida en que este ethos de la libertad es también una manera de ocuparse de los otros... Nos encontramos así también con el arte de gobernar. El ethos implica también una relación para con los otros, en la medida en que el cuidado de sí convierte a quien lo posee en alguien capaz de ocupar en la 
ciudad, en la comunidad o en las relaciones interindividuales el lugar que conviene -ya sea para ejercer una magistratura o para establecer relaciones de amistad- Y, además, el cuidado de sí implica también una relación con el otro en la medida que, para ocuparse bien de sí, es preciso escuchar las lecciones de un maestro, uno tiene necesidad de un guía, de un consejero, de un amigo, de alguien que nos diga la verdad. De este modo el problema de las relaciones con los demás está presente a lo largo de todo este desarrollo del cuidado de sí (Foucault, 1994, p. 116).

Esto nos permite también entender el concepto de cuidado de sí también conectado a un contexto cultural, económico y político. El que cuida de sí mismo sabe cuáles son sus deberes. Sin embargo, se conecta con sus deberes en la medida en que cuida su propio yo y su individualidad.

\section{Las competencias en perspectiva crítica}

La pregunta sigue siendo la misma desde el principio «¿Qué tipo de ser humano y profesional pretendemos formar, en un contexto cambiante?» (Arias, 2009, p.70). Y es un error pensar que cada tendencia que interpreta las competencias es neutral con respecto a la manera cómo se ve el ser humano. Por ejemplo, la tendencia económica política ve al ser humano desde el punto de vista del darwinismo social, como un medio para la consecución de fines económicos, como «una pieza más del engranaje económico y social» (Arias, 2009, p. 71). Si pensamos en este hecho, se nos hace difícil pensar en que concepciones diferentes de la persona puedan conciliarse hasta el punto que una pueda servir de contrapeso a la otra. Es cierto que desde la perspectiva del maestro el objetivo es buscar una mirada integral y que desde ese punto de vista el maestro debe atender a tanto a las exigencias políticas educativas como a la vida misma de los estudiantes y su individualidad. Pero żqué hacer cuando estas dos cosas entran en conflicto, cuando las exigencias políticas educativas tienen como propósito, como parece en Colombia, integrar a los seres humanos en un ámbito laboral productivo sin atender a sus necesidades individuales?

Lo que yo quisiera ver en el concepto de cuidarse a sí mismo que expuse usando la filosofía de Foucault es una idea general que nos obliga a reconocer un ámbito del ser humano que va más allá de cualquier saber y cualquier destreza práctica. Lo más importante en este ámbito no es 
una esencia predefinida de ser humano, sino el hecho general que el ser humanos construye su propia esencia a partir del respeto a su individualidad. Para este propósito la autorreflexión no debe entenderse como autorregulación a partir de estándares y criterios preconcebidos sino como una exploración del individuo y sus capacidades más allá de su contexto socio económico. Desde este punto de vista, la noción de cuidado de sí va más allá de la homogeneización de los sujetos provocada por currículos que atienden a criterios del mercado. La tendencia económica desde este punto de vista parece seguir la idea de la relación entre sujeto y verdad ofrecida por el cristianismo que hacía necesario la renuncia del sujeto a sí mismo para alcanzar la verdad (Foucault, 1994, p. 90).

En cambio, esta idea de una pedagogía basada en la comprensión de las competencias a partir del cuidado de sí, tiene como consecuencia que no necesariamente debe haber una renuncia a sí mismo en el sentido de autorregulación, sino que el propósito es generar en la y el sujeto un amor hacia su propio yo y hacia el conocimiento que desea adquirir. En caso contrario estaríamos promoviendo una nueva forma de esclavismo que tiene como propósito el trabajo básico. Pues educar es también educar para gobernar, para el gobierno de sí mismos y de los otros (Foucault, 1994, p. 116).

La labor de los currículos entonces debería apuntar hacia el ser de las personas, que la persona logre alcanzar su ser, que logre desarrollar su creatividad y sus potencialidades para que así él pueda empoderarse de su futuro, no atender pasivamente al futuro impuesto por el mercado. Esto no es ir en contra de las realidades económicas ni es olvidar las tendencias que hacen énfasis en el «saber qué» y en el «saber hacer». Lo que implica es simplemente organizar estos conceptos a partir de la reflexión sobre la individualidad del sujeto y el cuidado de sí en tanto idea rectora del concepto de competencia. Crear currículos a partir de este concepto de competencia implica asegurarse que los contenidos no sólo deben enseñar a hacer sino que deben inspirar y generar amor por el conocer. Este es un aspecto que no tiene en cuenta ni los currículos, ni los criterios de las competencias ni su evaluación. Algo que ha sido olvidado por las urgencias de nuestro mundo globalizado, y es algo que los maestros tenemos la responsabilidad de rescatar más allá de un discurso institucional vacío que pretende enmascarar una realidad distinta. 


\section{Conclusión}

La perspectiva presentada sobre la noción de o sus tendencias parte de un ideal de educación que requiere que sea pensado y hecho realidad, a través de la práctica educativa y a través de una concepción amplia del concepto rector de competencia. Antes que rechazar el concepto por estar vinculado con intereses productivos, el propósito fue abordar una de sus dimensiones y mostrar que el «cuidado de sí mismo» no reduce el concepto a puro conocimiento (ya sea de sí o de una cosa) o a un saber hacer productivo. Entender la idea de cuidar de sí mismo, con el apoyo del maestro(a) y la escuela, contribuye a recuperar la idea de educación y del conocimiento como una forma de amor a sí mismo, al saber, a los otros, y a la sociedad.

\section{Referencias}

Arias, N. (2009). Tendencias en la concepción de competencia, en la educación superior: una respuesta al interrogante. ¿Qué tipo de ser humano se pretende formar?. En Revista Educación y Desarrollo Social. Vol. 3. No 2

Foucault, M (1994). Hermenéutica del sujeto. Madrid: Endimión.

Ministerio de Educación Nacional de Colombia (2005). Competencias y reformas en la Educación Superior. En Boletín No. 5. Octubrediciembre. Recuperado de http://menweb.mineducacion.gov.co/ educacion_superior/numero_05/0001.htm.

Miinisterio de Educación Nacional de Colombia (2006). Documento sobre Estándares Básicos de Competencias. Recuperado de www. mineducacion.gov.co/1621/articles-116042_archivo_pdf.pdf.

Ruiz, S. (2004) En torno al concepto chosmkyano de competencia. Recuperado de http://www.uia.mx/campus/publicaciones/altertexto/ pdf/3ruiz.pdf.

Salas, W. (s.f.). Formación por competencias en Educación Superior. Una aproximación conceptual a propósito del caso colombiano. Recuperado de http://www.rieoei.org/deloslectores/1036Salas.pdf.

Zemelman, H. (2010). Lenguaje y producción de conocimiento en el pensamiento crítico. México DF: Cerezo Editores. 